\title{
7 \\ ENERGY: WHERE IT ALL BEGINS
}

\section{In which the relationships between energy and evolutionary change are followed from the first ancient cell to Gaia.}

The continuous flow of energy from the Sun is responsible for the maintenance and self-organisation of life on Earth. Cycles of energy establish the environmental conditions under which evolution can occur. We can follow the interrelationships between energy flow and evolutionary change from the very first cells to the complexity of the natural systems of today.

'The flow of energy through a system tends to organise it' is an aphorism that describes the world that we encounter every day. Provide human babies with adequate nutrition and, with the energy they derive from it, and within the possibilities of their genetic program, and with culturally appropriate care, they self-organise into adults. Here we address the processes of self-organisation of Gaia, and its many characteristics, that are brought about by the flow of energy from the Sun. These include the amount of time that energy spends circulating through the biosphere (its dwell time), how it engenders cycles of resources and how it supports increasing complexity. All this is achieved in the face of the tendency of things to run down, the tendency for disorder. Without energy flow, in the words of WB Yeats:

Things fall apart; the centre cannot hold;

Mere anarchy is loosed upon the world, 
Energy flow from the Sun is an absolute requirement for all life on this planet (with the exception of a number of ancient cell types that can use geothermal or chemical energy). The Earth absorbs the energy like a huge greenhouse, with an atmospheric shield of gases instead of glass. Inside the 'greenhouse' every animal depends ultimately on the ability of green plants and algae to turn carbon dioxide into sugars and release oxygen. This is the chemical process of photosynthesis that takes place in the small specialised organelles in the plant cell called chloroplasts whose ancestors were once, long ago, independent ancient cells. It is estimated that the humblest of ancient cells, the blue-green algae that comprise a major part of marine plankton, are still responsible for 20 per cent of the total photosynthetic output of all green plants. This by-product of photosynthesis, oxygen, leaks into the environment and maintains our stable, breathable atmosphere.

Figure 15 is a diagram of the Solar System as a steady state. The Sun radiates energy in all directions; some of that energy is 'captured' by the Earth. It flows through the biosphere and is finally radiated into space. As long as the entropy - let's think of entropy as disorder or chaos as long as the entropy of the whole system increases, it does not violate Newton's laws if a small corner of it - the biosphere - shows a decrease in entropy and becomes more ordered or complex.

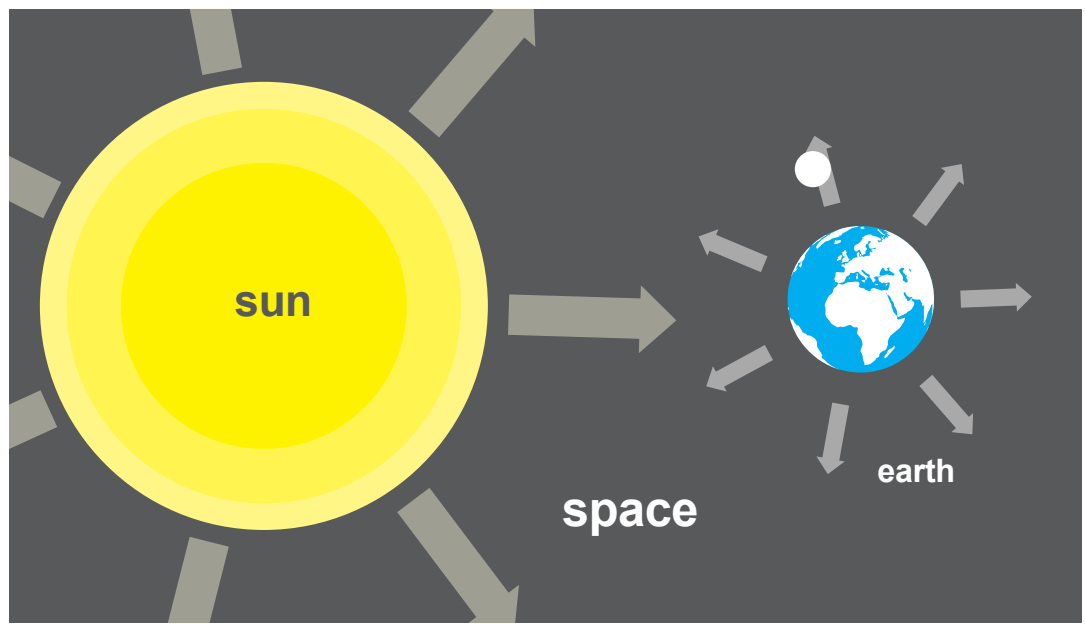

Figure 15. The flow of energy in the Solar System. 
The relationship between the Sun and the Earth is therefore one of energy flow and, from the short-lived point of view of living things on Earth, to all intents and purposes, it is a steady state, a condition that does not vary with time. Certainly the Sun's output has fluctuated, and will fluctuate, but the time scale for such fluctuations is measured over millions of years: from our point of view, that's steady.

Energy therefore flows from its source, the Sun, whose light shines on the photosynthetic green mantle of Earth. The sugars that are created by green plants are used immediately to sustain their own lives and growth. Any surplus is laid down in storage organs, such as roots and tubers, for future use. It may, however, be stolen by other organisms, including humans buying potatoes and onions at the greengrocer.

Plants and animals die and their remains become food to be converted into energy by other plants, animals, bacteria and fungi. After a little while, they also die and become humus, the dead organic matter on which future plant growth depends. Sometimes, when environmental conditions permit, once-living organic matter is fossilised as coal and oil deposits. Finally, excess energy, as heat, is radiated into the unfillable 'sink' called space. The amount of time that energy spends moving around Gaia is called its 'dwell time'. It is the time that the Sun's energy may be available for use by living things.

In spite of the efforts portrayed in numerous science fiction stories, it is not possible to imagine where the sum of evolutionary change will take us under these circumstances, even 100 years hence. For one reason, ever since the discovery of fire, an important component of the total human energy budget has been provided by fossil fuels laid down millions of years ago. We do not know, though we can make informed guesses, the form of energy that we will need in the future. The present choices, as we turn away from fossil fuels and toward renewables, seem to be nuclear fission, solar, wind, hydroelectric, geothermal, tidal or other uses of the omnipresent gravity. We do know that energy will be needed, however we decide to harness its flow.

We can best understand what can be accomplished with energy flow systems by considering the many applications of the concept of flow in human societies. For example, one of the most important innovations of Roman civilisation was their engineering of water flow and the treatment of sewage. The Romans probably take the credit for creating the first 
system of drains. They conceived of them as simple removal devices, to transport unwanted waste products somewhere else. They were not concerned with sewage treatment per se - they wanted to get rid of the smell, the 'miasma' that they thought was responsible for disease.

A modern sewage system involves treating the sewage and recycling the safe organic material that is produced. In efficient systems, from early agriculture to major cities, sewage material has been allowed to act as recycled energy. Medieval farmers knew this well, as their livelihood depended on their dungheap. In a well-organised sewage system, the dwell time - the storage time between the origin of the waste and the use of the effluent - is very important. Flow must be continuous and constant. If the sewage is allowed to build up, it will overflow in inconvenient places. If there is no flow through the system, it will stagnate and provide an energy source only for bacteria. Ideally, in a modern sewage farm, the rate of flow allows useful biological systems to operate; the heat they generate during dwell time eliminates the disease-carrying organisms and allows the formation of the energy-carrying gas, methane, which can be stored for immediate or later use.

Consider another flow system, common in medieval times, the waterdriven flourmill. It was typically set close by a stream, at a point on the watercourse where the water coming from higher up had sufficient mass and velocity to do work as it fell downhill. By inserting a millwheel into the stream, the miller was able tap its energy to turn the wheel and hence turn the grindstone to mill the flour. The volume of flow was very important. If too little water passed down the stream it would not have had sufficient energy to turn the wheel. To guard against this eventuality in times of low rainfall, watermills have millponds above the wheel to collect and store as much water as possible during wet periods. By operating a sluice the miller can direct water from the pond into his millrace that channels the water past his wheel. For the miller, ideally, the dwell time for storing water in his pool should be as long as possible. He wants to be able to rely on a constant, effective flow to mill his grain.

These two examples demonstrate that in dynamic flow systems, like sewage disposal and milling, both rate of flow and dwell time are important. These ideas create a metaphor repeatedly encountered in human affairs. We talk of the flow of time, of music, of poetry - and of the wealth that makes all the aforementioned possible. Economists have a maxim that 'the flow of money through a system (a company, a village, 
a town, a city, a country) tends to organise it'. That is, it increases its structural complexity (see Fruth 2015). Rate of flow of money is obviously important; too little and an organisation shrinks as jobs are lost.

As employees, people represent money. The decrease in the rural population in England during the Industrial Revolution was due to farm hands migrating to the cities, where the 'dark satanic mills' of Blake were to be found. The flow of people was equivalent to an outflow of money from the farming sector to the wool and cotton industries. The remaining workforce was no longer sufficient to support the existing agrarian infrastructure. Even worse, in the society built around the Industrial Revolution, the dwell time of money was inadequate to allow much development for the working community. It flowed straight into the pockets of the great mill, mine and landowners who had other uses for it. More recently, as more complex flows developed, people have developed structures - cooperative societies, schools, hospitals - to increase the dwell time of money and address their different needs.

The flow of solar energy from the Sun, through the Earth and back into space is the embodiment of Newton's laws of thermodynamics reworked as Carnot's principle (see, for example, Laranjeiras and Portela 2016). Heat always flows from a hotter body to a cooler body. It was implanted indelibly into young minds nearly 60 years ago by one of the songs of Flanders and Swann that began, 'Oh, you can't pass heat from the cooler to the hotter'. (To underline the power of such a mnemonic in teaching children, one of us has never forgotten that the operating cycle of the internal combustion engine is 'suck, squash, bang, blow'!)

During the process of energy flow from hotter to cooler, Newton's second law demands that entropy, the amount of disorder in the whole system, must increase - remember, 'things fall apart'. Entropy is a technical word but perhaps it is easier to think of it as the universal and continuing breakdown of structure. Energy collected from the Earth's self-organising systems is continually flowing outward, as heat, into space. It follows that a highly organised system, like a human being, with lots of order created out of information contained in DNA, is relatively low in entropy (disorder). In creationist literature, it is often remarked that, because order increases and entropy decreases in living organisms, rather than the other way round, the second law does not apply to them. This, they claim, somehow sets living things outside of normal thermodynamic considerations. This thinking demonstrates a fundamental misunderstanding of energy flow 
principles. In steady state systems as long as the entropy (disorder) in the whole system increases, then, it is quite allowable for small parts of it to show less disorder. This is the reason for the entropy decrease achieved by Gaia within the background of the increasing entropy of the Solar System. The small planet uses the dwell time of the energy for the selforganisation of living systems. Figure 16 illustrates the great carbon cycle that drives Gaia.

If the Earth were a homogeneous, smooth ball of rock, it would warm up uniformly and cool uniformly. The real Earth is very different, it is composed of trillions of objects, some of which warm up and cool down more quickly than others. In addition, the Earth rotates, presenting a continually different surface to the Sun. Instead of uniform warming it is patchy, as the hottest objects pass heat to the more cool ones. All these interactions combine to increase the dwell time of energy on the surface of the Earth, giving it time to do work. Green cells and plants are among these trillions of objects and they capture energy and process it in very small, manageable parcels indeed.

Perhaps an analogy will make this clearer. The Sun pours out energy unremittingly. When it hits the Earth the only thing that happens in the absence of life is that everything gets extremely hot and then cools down as the Earth turns, rather like conditions on the Moon. This is what is meant by a vertical temperature gradient.

Let us now suppose that the flow of energy is like the flow of a great river. Imagine this river going over a waterfall with a 100-metre drop. The potential energy of the water as it flows over the edge is very great but much of it is dissipated at once when it hits the ground, gouging out a great pool at the bottom in a cloud of spray, and then flowing on its way. The fall of water is also a vertical gradient.

Now, instead of the water dropping 100 metres, imagine that it is flowing down a staircase of 100 steps, each with a height and width of one metre. The vertical gradient has been replaced by one of 45 degrees. The energy lost by a 1-metre fall is not very great at each step, but 100 steps, one after the other, adds up to a great deal of energy indeed.

It is now time to reintroduce the water-driven mill and a miller who mills flour. He's a resourceful chap who knows about gradients. He wants to harness the energy of falling water to turn his grindstone. He immediately rejects the idea of trying to tame a waterfall because the vertical gradient 
- the long drop - produces more energy than he can reasonably handle; his millwheel would be reduced to matchwood. He looks for a shallower gradient along the river, and very soon discovers a promising site. But it's still quite a large river just there and he imagines what it must be like in the wet season, in full spate, probably bringing small boulders down that will damage the millwheel he is planning to build. He therefore decides to position his wheel some way from the river. So, from a point upstream, he constructs a small canal and a sluice gate that collects water and discharges it into the large pond - the mill pond - that he has dug ready. By opening a sluice gate in the pond he can release water into a channel that runs right by his mill where he intends to insert a millwheel. The water will turn the wheel that turns a grindstone that will grind his corn and he's in business. The water has less energy as it leaves the millwheel, but that is still a lot of energy going to waste if someone wants to tap it. Being an ingenious fellow, he develops a clever system of gearing to trap some of the surplus energy from grinding flour and adds a potter's wheel and, later, a lathe. $\mathrm{He}$ can now make and sell kitchenware and furniture as well grinding flour for the local village.

And that is analogous to the thing that all living green plants do. They capture some of the energy from the Sun and use it drive a series of small chemical steps, a metabolic pathway, to make simple sugar molecules.

Life thus deals with the vertical drop of the thermodynamic gradient from the Sun to the Earth by converting it into a 'descending staircase' with many chemical steps. Each step represents one tiny, manageable bit of energy. A plant captures energy with its chloroplasts and uses it, by a series of reactions, a metabolic pathway, to make sugars and starch. These might be stored in specialised organs, such as potato tubers or daffodil bulbs. When energy is required for respiration and growth by the plants, another pathway is brought into play and starch is first turned into glucose, that is then oxidised (or 'burned' as a marathon runner 'burns' carbohydrates) to provide the necessary energy. During the process glucose gives up its energy in very small parcels, rather than all at once. This is just as well, because if it did give it all up at once it would go off like a firework and cook the cells in which it was happening. Starch dust so readily generates heat when it is breaking down that sometimes it blows up wheat silos.

An industrial power station does essentially the same thing as the local miller. The power station burns coal to carbon dioxide and water and energy is captured and converted to a different form, electricity, for use. 


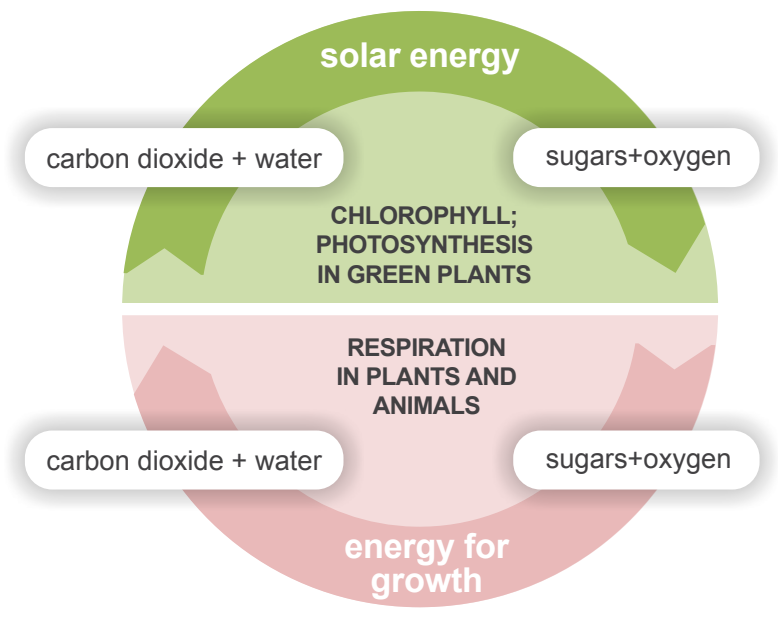

Figure 16. Gaia's carbon cycle.

The great energy cycle, the carbon cycle of Gaia, that supports all life on Earth is thus completed. What has happened is this:

1. Energy capture from the Sun by green plants and blue-green algae:

- carbon dioxide and water are converted by photosynthesis to simple sugars, and oxygen is released. (This is where all the oxygen in the atmosphere comes from.)

2. Respiration by all living things - animals, green plants, fungi and ancient cells:

- sugars are oxidised by oxygen and converted to carbon dioxide and water.

Very little observation will demonstrate that living things increase in complexity during evolution, from ancient microbes to modern blue whales. Increasing size and complexity are only possible because energy flows through the biosphere. Complex systems are maintained by energy flow, so it is obvious that achieving greater complexity uses up greater amounts of energy. The limit to complexity is defined by the maximum amount of solar radiation consistent with the continuation of life on Earth. We cannot know what point on this upward curve of complexity we have reached. It seems possible that the rate of increase in complexity is slowing. This does not mean that the overall rate of evolution is necessarily slowing, as we must also presume that it continues simultaneously at all levels of organisation from the single-celled microbe to multi-celled blue whales. 
If energy flow generated by green plants leads to greater and greater complexity, it must also lead to increasing instability. More energy capture is required for maintaining increased complexity.

As life gets 'pumped up' to higher and higher levels of complexity and connectivity, it still remains hostage to chance. Asteroids colliding with Earth, or a period of increased volcanic activity can raise dust and vapour clouds that reduce the amount of the solar radiation that reaches the surface of the Earth. In the past, these have resulted in wholesale extinctions. Six major extinctions are on record since the earliest emergence of life. Another possible candidate for the status of great extinction is the current stripping of natural habitats by humans. On the upside, an extinction provides great opportunity for evolutionary change, since there are then a multitude of potential environmental niches left vacant.

Lovelock must welcome the modern emphasis on the role of niche improvement in evolution, since his Gaia hypothesis is predicated on just this concept. Niche improvement goes on all around us all the time. Any gardener will tell that you that planting potatoes improves the soil by breaking it up and improving drainage. The gardener will then take advantage of it by planting other vegetables in the bed the following year. It is a trivial example, perhaps, but now think about rainforests, which, over thousands of years of evolution, have fixed carbon dioxide into leaves that fall and decay, creating humus that was not there before the rainforest. Or birds that build elaborate nests that permit the more efficient incubation of eggs; or the nests of wasps, the hives of bees, the mounds of termites. Or the first green plants to invade the land with their probing roots that first assisted weathering of the rocks and later, much later, stabilised the valuable soils that came into existence or Homo sapiens, the greatest niche 'improver' of all animals. Think of the global cities, households, hospitals, schools. All of these social constructions require reliable flows of energy to establish, maintain and improve the Homo sapiens niche.

It is all part of a great, if unintentional, collaboration. The changed environments of each niche are hotbeds of natural selection. An improved niche is a territory in which the mix of energy flow, geochemistry, microclimate and coexisting biology becomes capable of supporting forms of life beyond that for which it was created. In the same geographic space that contains, say, an ant's nest, there are countless other niches that are occupied by non-ants: plants and animals that peacefully coexist with 
the ants. They would not exist had it not been for the ants improving their own fitness to survive by building the nest. All of them interact and are subtly changed by the interaction. Leonardo da Vinci wrote: 'realize that everything connects to everything else'. Gaia is a great all-inclusive network of niches. Together, niches form a huge and intricate threedimensional mosaic that sits on the molten core of the Earth.

It seems possible that modern society is approaching a crisis point in its handling of energy, too clever by half in handling its own niche improvement. Unlike the ants, humans can visualise in advance ways in which they might work to improve their lot. No ant ever made a wooden armchair, although termites, no doubt, have eaten many!

As the human population grows, energy flow from the Sun will remain relatively stable. The world's ecosystems, however, are being exploited at an ever-increasing rate. The World Wildlife Federation has recently warned that, by the middle of this century, humans will need the equivalent of two planet Earths to continue to live as we are at present. Simply put, we are using the planet's resources more quickly than they renew themselves. If there is less to go around, standards of living must fall or else human populations become ever more sharply divided into the haves and havenots. Australia is a test case. We have limited resources and what we do have a lot of, like iron ore and coal, other countries are wanting less and less. The human carrying capacity of Australia is limited by the availability of water and generally poor soils. Some authorities suggest that a human population in Australia of between 50 and 100 million is the limit without vastly improved techniques for agriculture and renewable energy use, and much greater investment in infrastructure. The real point is this: in a steady state solar system, energy flow is finite and not all the energy is available for use. One has always to pay one's dues to counter the tendency to disorder. Or, to put the relationship between Earth's energy balance and increasing entropy in the universe in terms that a gambler could understand (popularly, if inaccurately, known as Ginsberg's theorem):

You can't win.

You can't break even.

You can't quit the game.

Whatever happens, you lose. 
Gaia has three important characteristics that are essential for her continued existence, all of them involving constant energy flows. They are: first, great complexity and a high level of order; second, feedback and feed-forward cycles that regulate the whole system to give stability and longevity; and third, cycles of cycles - hypercycles. They are exemplified at the cellular level, in biochemical pathways that cycle; at the organismal level by the many interacting reproductive cycles; and at the environmental level by the carbon and nitrogen cycles. The planet itself cycles around the Sun.

Cycles underlie the whole of human existence. Humans are conceived, increase in complexity until they reach maturity, a steady state, lasting perhaps 30 or 40 years, and they contain many biochemical and physiological cycles. Humans, in fact, are rather like the blacksmith's hammer, the one that belonged to his great-great-great-grandfather. The shaft has been replaced half a dozen times, and it has had a few replacement heads, but it's still the same hammer - isn't it? All human cell types undergo renewal cycles at different rates, except the cells of the cerebral cortex, which are not renewed, although they can be reprogrammed. But you are still the same person - aren't you? After a time the steady state decays and senescence and death follow, when all human order is lost, and its remains are returned to the ground to participate in other cycles.

The evolutionary history of life on Earth is a history of the complexity and order of life, as they increase with geological time. We have agreed that as complexity increases so does the need for energy to maintain it. An ancient cell is less complex than an independent modern cell. These are less complex than multicellular organisms like sponges. Sponges are less complex than elephants. But it is important to remember that bacteria, protozoa, sponges and elephants are all with us today - and what is more, ultimately, we are all related, in that we share an ancient common ancestor. Each level of organisation does not therefore replace the previous one, rather it builds on what has gone before so that the whole of life becomes an interconnected, layered phenomenon.

To travel, in your mind, the path that leads from the simplest cell to the mind-boggling blue whale is to undertake a journey of continual wonder. Twice before we have referred to the shocking discovery of the apparent small size of the human genome and remarked how astonishing it is that it comprises only about 20,000-odd genes. Even a single-celled soil amoeba has almost 16,000, while a tomato plant has more than 30,000. Many of these genes are highly conserved; that is to say, they represent 
combinations of genes that remain responsible for the most fundamental processes of life. The fact that they are present in all animals, plants and fungi allows us to determine degrees of relatedness. From this we learn that we all speak the same genetic language and, as we have noted, DNA is a celestial Lego set, a vast collection of building blocks that are used over and over again in different combinations to create the many forms of life.

The Earth is about 4.5 billion years old. Some of the oldest fossils are found in rocks that are about 3.5 billion years old. They are thought to have been primitive cells (cyanobacteria, also called blue-green algae) that obviously must have had their beginnings well before that time. There are various scenarios that attempt to describe how the world before life brought forth the first 'protocells' that were their ancestors, but they all agree that a continuous flow of energy such as that from the Sun created the necessary thermodynamic preconditions. There are many scenarios that attempt to explain what happened next and a recent book by Ward and Kirschvink (2015) gives an excellent account of all of them.

Viruses are the first step in the journey to the blue whale. Recent discoveries indicate that the virus is, as it were, part of us. This is both scientifically and aesthetically pleasing, as it emphasises yet again that we are all linked into Gaia and we no longer might have to make exceptions for viruses. Perhaps it is not so surprising after all, as viral DNA is frequently found inserted into in the genomes of higher organisms and we can now legitimately embrace it as a source of evolutionary novelty.

On Earth, wherever there is an accessible energy source, a life form has taken advantage of it, and evolved from there. The discovery of volcanic vents in the deeps of the ocean provides the fuel for a number of 'origin of life' postulates. 'Black smokers' (Tunnicliffe 1991) attract attention because they create environments equipped with the necessary chemical set for early life and in which dwell representatives of the earliest living forms on Earth. Cooperative biology starts here!

It has even been suggested that, if recent evidence of ancient volcanic activity on Mars is correct, life may have had its origin there. Calculations that Mars rocks, thrown out by volcanoes, could reach escape velocity and fall to Earth offer the beguiling prospect that we are all Martians. Some billions of tonnes of Mars rock are supposed to have made this journey so far. Perhaps one was sheltering a primitive Martian molecule that won the jackpot by ending up near a vent. 
However it happened, the first protocell (a cell that is not yet quite a cell) that appeared was essentially a membrane containing a large number of functioning chemical units (molecules). The formation of a boundary membrane was an absolutely critical step in organic evolution. Only by containing these molecules within a bounded environment in effectively high concentrations, together with an energy source, could there be any internal control. Homeostasis, that is to say, regulation of the composition of the protocell within limits defined by being alive, was at last possible. The final important step was the acquisition of an almost perfect mechanism for cell division. It must have been almost perfect because a perfect mechanism would always result in identical daughter cells. Subsequent evolution would then have to depend on external events, such as irradiation, to produce variation and to allow natural selection. Darwinian selection hangs on the fact that offspring vary, so the almost perfect system sets up the preconditions for evolution to take place.

Today, all the Protista (protozoa and single-celled algae), fungi, plants and animals are organisms made of modern cells. While we humans are also comprised of modern cells, it must be remembered that many other ancient cells are found within our intestines and upon our skins. These are our partners and most of them contribute to our wellbeing. Others, relatively few, are parasites that attack us and contribute to our illnesses and our mortality. We are actually doing pretty well because, according to one estimate, the world contains about 5 million trillion trillion ( 5 followed by 30 zeroes) ancient cells. This works out at a biomass of about 1,000 times the total weight of the human biomass in the world. Approximately!

Early attempts to describe modern cells as the result of symbiosis between earlier cells were laughed aside. It was no surprise, then, that the Margulis hypothesis that modern cells were made up of a collaboration between three or four ancient cells met with frank disbelief at first. The implications of the Margulis hypothesis, however, are enormous. Three billion years ago the Earth belonged to the ancient cells. Competition between them must have been intense. Cells had preyed on or parasitised other cells. Adaptations of offense were countered by ones of defence. While many cells must have succumbed to attack, many others managed to coexist with their attackers. But some billions of years ago a third type, working in intimate cooperation with other cells, had managed to establish many symbiotic associations, to be tested by natural selection. In support of this view, very recently, an intriguing new type of organism has been 
discovered which appears to be an intermediate form - a missing link! between an ancient cell and a modern cell. This missing link, Parakaryon myojinensis, if confirmed, is compelling evidence for the capacity of cells to form a variety of cooperative relationships (Yamaguchi et al. 2012).

Every multicellular organism, including humans, is comprised of modern cells. On top of this, humans have a relationship with 500-1,000, at least, types of ancient cells, billions of bacteria living in the intestine and elsewhere. Bacterial cells are so much smaller than modern cells, and it has been estimated that, in the average human, there are three times as many microbial cells as modern ones. A human is truly a biological community.

And here is a thought. Among the earliest evidences of life on Earth, more than 3 billion years ago, are fossilised stromatolites. They are structures comprising microbial 'mats', especially blue-green algae, bound together in layers by concretions of sand grains. They grow as columns in warm shallow waters. A visit to Shark Bay in Western Australia is well worthwhile, for anyone interested, to see modern examples. The stromatolites are actually a scaffold on which these ancient cells could thrive in the light. The thought? Perhaps humans, from the point of view of their internal ancient companions, should be regarded as mobile stromatolites!

This chapter has moved from the stark realities imposed by the physical laws of thermodynamics to a consideration of the biophysical laws of energy flow and the 'steady state'. The apparently inevitable natural synthesis of the molecules on which life depends, followed by self-organisation to produce the first ancient cells, was followed by the most momentous event in evolutionary history, the collaborative symbiotic origin of the modern cell. Forms of life interacted cooperatively at the first possible opportunity. This created a precedent that continues to this day and has been going on for billions of years - perhaps even longer.

Humanity started its own journey to harness energy with the discovery of the uses of fire only 1 or perhaps 2 million years ago. The discovery that fire could be controlled led inexorably across the 2 million years by way of primitive technologies of the stone, bronze and iron ages to the Industrial Revolution. Along the way, the energy in wind and water has been also harvested, and tidal energies have been tapped.

The discovery of radioactivity opened doors that many think might better have remained closed. However, once a door is open it does not seem to be within the power of humanity to close it again - at least, not without 
first finding out what is on the other side. So far, the atomic bomb has been used in war on only two occasions and now, surprisingly, atomic power stations are in decline, not due to ethical considerations but to obsolescence and the lack of profitability in building new ones. This unexpected outcome is discussed in 'Nuclear Holiday' by Lisa Grossman (2017). Perhaps an era of utilisation of renewable energy sources beckons until fusion - using the same reaction that occurs in the Sun - becomes a viable alternative.

The chapter has pursued the many ways in which energy supply and evolutionary pressures go hand in hand. These include the length of time that energy can be stored (dwell time), the expansion of ecological niches, the cycles of resources that together make up Gaia, the universal tendency to disorder and the complexity of self-organising systems. Each of these are fertile sources of difference, and with difference comes the evolutionary opportunity.

The next chapter is concerned with the ubiquity of the evidence of cooperation between different forms of living matter. It starts from the point that parasitologists have long been aware of. There is no known single species of organism that does not have another one living on, in or with it. It moves on through evolutionary history to reach the evolution of the social-based system that is humanity. 
This text is taken from Cooperative Evolution: Reclaiming Darwin's Vision, by Christopher Bryant and Valerie A. Brown, published 2021 by ANU Press, The Australian National University, Canberra, Australia.

doi.org/10.22459/CE.2021.07 